\title{
Pre-Slaughter Stress, Management of Stress and Its Effect on Meat and Carcass Quality
}

\author{
Addis Fikrie Birhanu \\ Meat Product Research and Processing Directorate, Ethiopian Meat and Dairy Industry Development Institute, Bishoftu, Ethiopia
}

How to cite this paper: Birhanu, A. F.(2020) Pre-slaughter Stress, Management of stress and its effect on Meat and Carcass quality. International Journal of the Science of Food and Agriculture, 4(1), 30-37.

DOI: $10.26855 /$ ijfsa.2020.03.006

Received: December 5, 2019

Accepted: January 3, 2020

Published: February 12, 2020

*Corresponding author: Addis Fikrie Birhanu, Meat Product Research and Processing Directorate., Bishoftu, Ethiopia.

Email: addsfkr@gmail.com

\begin{abstract}
Pre-slaughter stress includes all the conditions and practices that apply during the period when the animal is moved from source or market to enter to the stunning box at the abattoir. Cattle can be stressed during transportation, marketing and handling at abattoirs. During such condition physiological changes (increased heart rate, respiratory rate, body temperature, the activity of enzymes, stress hormones (catecholamine, cortisol and glycolysis) have been occurred which used to measure stress before cattle slaughter. Such changes affect the meat quality by depleting muscle glycogen which results in a lower rate of post-mortem lactic acid synthesis, high ultimate $\mathrm{pH}$, tough meat, abnormal muscle color and high water retention capacity. Pre-slaughter stress also causes carcass damage (bruising, hemorrhages, and blood splash), low carcass yields, and total carcass loss meat. Pre-slaughter dietary supplement (Magnesium, Chromium Tryptophan, electrolyte, and Vitamin E), appropriate livestock facilities, best animal handling practice and selection for temperament have been the most effective way to reduce pre-slaughter stress.
\end{abstract}

\section{Keywords}

Pre-Slaughter Stress, Quality, Meat, Carcass, Physiological

\section{Introduction}

The transformation of slaughter animals into meat is a chain of events including handling, loading, transporting, unloading, holding and finally slaughtering. During all these activities due poor handling, operational techniques, and facilities cattle exposed to various stress condition such as noise, vibration, restraint, deprivation of feed and water, adverse weather conditions, breakdown of social grouping, mixing with unfamiliar animal, overcrowding, increased human contact and improper stunning before slaughter which lead to unnecessary suffering, injury and loss of production $[1,2]$

Stress defined as a physiological disturbance that is linked to mental states associated with threatening or harmful and painful situations [3]. Pre-slaughter stress can be differentiated into physical and psychological stresses [4]. Physical stress can be caused by high ambient temperatures, vehicle shake, noise and density, slippery floor, lack of food and water as well as loading and unloading from the truck [5,6]. Psychological stress can be caused by group separation, mixing with an unknown group, new environment, handling and increased human contact [7]

Such stress condition may lead to some changes in concentration of enzymes (creatine kinase, lactate dehydrogenase, aspartate aminotransferase ,alanine Aminotransferas, alkaline phosphatase ), hormone (catecholamines, cortisol) and other blood constituents such glucose and packed cell volume blood [8] which 
result in glycogen depletion causing lower rate of post-mortem lactic acid synthesis, high ultimate $\mathrm{pH}$, undesirable color, and greater water holding capacity [5]. Moreover, it causes carcass damage through bruising, hemorrhages, blood splash to the extent of a total loss of carcass and reduction in live weight [7]. Stress detection of animals before slaughter is needed to help the management of animals in order to produce a good quality of meat.

There was no well-documented information on pre-slaughter stress, its measurement, and impacts on meat and carcass quality. Therefore the object of this manuscript to review cattle pre-slaughter stress, management of stress and its impact on meat and carcass quality.

\section{Animal Welfare}

Concern for animal welfare is a major consideration in meat production based upon the belief that animals can suffer. Meat consumers are increasingly demanding that animals be reared, handled, transported and slaughtered using humane practices [9]. According to the Farm Animal Welfare Council [10], the welfare of an animal includes physical (an animal is fit and healthy) and mental state (animal feeling) and animal kept by humans should be protected from unnecessary suffering. Accordingly, the five freedoms have been a fundamental basis for animal welfare all over the world) (1) freedom from hunger and thirst: by providing constant access to freshwater and a diet to maintain full health and vigor; (2) freedom from discomfort: by providing an appropriate environment including shelter and a comfortable resting area; (3) freedom from pain, injury, or disease: by prevention or rapid diagnosis and treatment; (4) freedom to express normal behavior: by providing sufficient space, proper facilities, and company of the animal's own kind; (5) freedom from fear and distress: by ensuring conditions and treatment which avoid mental suffering.

Animal welfare defined as the state of an animal as it attempts to cope, both physically and mentally, with its environment [11]. Failure to cope can result in stress, described as the state of animal incapable to adapt, behaviorally and physiologically, to environmental or physical challenges [12]. Stress affects the animal's ability to cope, reduce its fitness and has negative implications on animal welfare. During stress, animals are prone to weight loss, produce poor meat quality and considerably reduced the profit of the producer [7].

\section{Stress Associated with Marketing and Transportation}

Animals are either sold at the markets or sent directly from farms to abattoirs for slaughter. Loading, transport and unloading conditions depend on the facilities on the farm, market and at the abattoir, and on the layout of the truck, the driving condition, and distance traveled. Transporting and marketing process is stressful to animals which involves removal from their home environment, loading and unloading onto vehicles, often long journeys and holding in unfamiliar surroundings. These results in their being potentially exposed to physical stresses such as extremes of temperature, humidity, vehicle vibration and changes in acceleration, noise, and confinement and crowding. There are also psychological or emotional stresses such as handling, breakdown of social groups and mixing with unfamiliar animals, unfamiliar or noxious smells and novel environments [7]. Animals may suffer from hunger, thirst, and fatigue because they are not usually fed before and during transport, and water may not be available. Other conditions such as injury, suffocation, and death may prevail in transportation [9]. Livestock transport in Ethiopia was done either by trekking, ordinary truck, and combination of both. In all methods of transportation animals are exposed to different stress [13]. Many studies have shown that loading and unloading are the most stressful part of the transport. The stress associated with handling at the time of loading and unloading can vary according to factors such as the quality of handling (gentle vs rough), experience of the handler, the temperament and condition of the animals and the quality of the handling facilities [14]. Loading is the most stressful operation than unloading because loading requires close proximity to humans and this can cause fear in animals that are not habituated to human contact and finally, pain may result from mishandling of animals (beating or poking animals with a stick, especially in sensitive areas like the eyes, mouth, ano-genital regions or belly): [15]

Transportation stress has shown to cause suppression of the immune system, making animals more vulnerable to disease. Cattle have been observed to have higher heart rates during transportation, which may indicate higher levels of stress, but may have also been due to increased activity during loading and unloading and from movement and exertion to maintain balance on the truck. Transportation has been also shown to cause elevated body temperature, which may have been due to psychological stress, physical activity or the increased internal 
temperature of the truck [16]. Cattle have exhibited other physiological indicators of stress during transit including increased creatine kinase concentrations indicating possible muscle damage and poor meat quality caused by stress markers such as high glucose and lactate concentrations [17].

Factors such as transportation time, stocking density, standard of driving, road condition, and design of the vehicle (size, space, and surface) are important in inducing pre-slaughter stress [18]. During transportation, animals can do little to help themselves when they are in danger or discomfort due to confinement. In farms, markets and lairage, animals needs much freedom of movement and could avoid certain uncomfortable conditions [19]. Over speeding, sudden stops, rapid acceleration and long journey times without appropriate rest should be avoided during transportation as these will increase carcass and meat quality defects. Careful loading, unloading needed to be practice and transportation to be done in a gentle manner under a quieter environmental condition to ensure the safety of animals in transportation to reduce stress to its minimum [20].

\section{Stress at Abattoirs}

At arrival at the slaughterhouse animals are unloaded, may moved for weighing, hold in reception pen until transferred to lairage. [21] Reported that moving cattle in abattoirs exposes the animals to a different structural design. The main purpose of lairage is to maintain a reservoir of animals so that the processing line in the abattoir can operate at a more or less constant speed irrespective of variations in the delivery of livestock. The second function is to allow the animal sufficient time to recover from the stressful effects of transport in order to restore their muscle glycogen levels, thereby allowing normal anaerobic muscle metabolism after slaughter that ensures good quality meat and better animal welfare [22]. However, lairage may be characterized by unfamiliar conditions, such as concrete floors as opposed to the natural grass farm, rough handling procedures, feed restriction, overcrowding, changes in the social structure (through separation and mixing), and the presence of unfamiliar animals which leads to stress condition [23].

High stocking density in lairage pens may promote fighting behavior in animals. Careless and improper handling of animals in the lairage such as the use of electrical goads, beating by sticks or rope and tail twisting are stressful to animals [22]. In addition improper stunning can also be stressful in which stunning tools do not function properly and/or poorly trained operators [6]. Abattoirs may have inappropriate lighting and many of them are very noisy [20]. Therefore providing cattle with an adequate amount of suitable feed and water if they will be kept longer than expected to promote glycogen accumulation, hydration, and calm behavior. Cattle should not be mixed with other unfamiliar cattle so that fighting does not occur [24]. Currently, different technologies such as ventilation equipment in lairage, appropriate lighting, limited use of electric goads and appropriate passageways for animals to walk have been used in abattoirs to reduce the stress in animals [25].

\section{The Nature of Stress Response}

Animal normally responds to stress with physiological and behavioral changes in a characteristic way. This stress response has two components. The first is a rapid short term alarm' response (defensive response). The acute stress response activates when the animal is exposed to a stress-stimuli for a short time. It contains either the flight or fight response. The preparations response involve the secretion of catecholamine hormones, adrenaline and noradrenalin (epinephrine and norepinephrine) and enzymes into blood circulatory system and eliciting a sequence of physiological responses, including increased heart and respiration rates, elevated body temperature and decrease protein degradation [9]. The second component of the stress response occurs after the 'alarm' response and over a longer time period (adaptive response). Its role is to allow the animal to recover from the alarm response or to 'adapt' to the new situation. This component of the animal's response to stress mainly involves the Hypothalamic-pituitary-adreno-cortical axis. During stressful episodes the hypothalamus secretes a hormone, corticotrophin-releasing factor (CRF). This stimulates the pituitary gland to secrete an adrenocorticotrophic hormone (ACTH) into the blood. ACTH in the blood stimulates the cortex of the adrenal gland to secrete corticosteroid hormones. The release of the corticosteroid hormones (cortisol) is a characteristic response of an animal to stressors [26,9]. Age, sex, health status, breed, and previous experiences of animals can influence the way they cope with stress [27].

\section{Measuring Pre-slaughter Stress}


When animals are exposed to pre slaughter stress conditions such as handling, transportation and environmental stressors (climate, temperature and nutrition), they react by eliciting certain physiological and behavioral changes in order to cope with the situation. These changes can be measured pre-slaughter to indicate how much stressed animals are suffering. Physiological changes such as increased heart rate, respiratory rate, body temperature activity of enzymes, stress hormones (catecholamine, cortisol), body temperature and glycolysis had been observed [28]. Enzymatic secretions which are released into the serum through leakage arising from altered membrane permeability include alkaline phosphate, acid phosphate, aspartate aminotransferase, creatine kinase and Lactate dehydrogenase [29]. These changes in enzymes and hormones indicate the level of stress created and reducing the quality of meat and its products. Hormone such as catecholamines (epinephrine and norepinephrine) are difficult and expensive to measure, not reliable as their concentration shows wide variation and their effects last only for a few minutes and only useful as indices of acute stress where samples can be taken very soon after the stress. Hence it is not the best indicators of stress [9].

\subsection{Creatine kinase and lactate dehydrogenase}

Creatine Kinase (CK) is an enzyme found in the skeletal muscles of animals, responsible for maintaining energy homeostasis at the sites of high ATP [30].This enzyme apears to be the most widely accepted and more generally used measure of stress sensitivity and the magnitude of stress associated with most systems of animal husbandry and pre-slaughter animal handling [31]. Rupture of muscles causes the release of CK which is deposited into the blood. The increased levels of CK in the blood (plasma or serum) are an indication of how stressful the handling was before the animal was slaughtered and the extent of muscular damage during handling and transportation in many species [32]. Moreover, the presence of this enzyme in the blood is due to breeding temperament, excitability and fighting [33].

Lactate dehydrogenase (LDH) is an enzyme found in almost all body tissues. It plays an important role in celllar respiration, the process by which glucose (sugar) from food is converted into usable energy for cells. This enzyme also increases in the blood after muscle and tissue damage [8]. Both CK and LDH have been used as an indicator of trauma, high levels of physical activity or other damages during handling and transport in farm animals.

\subsection{Cortisol}

Cortisol is a steroid hormone (glucocorticoid) released from the adrenal glands of cattle into blood during periods of stress and measurement of its concentration in blood had been reliable and widely used as a good indicator of stress [34]. Similarly [35] reported that increase in plasma concentration of cortisol has been used as an indicator of stress in cattle and widely used to evaluate the well-being of animals. Release of this hormone in times of stress resulting in an increase in blood sugar through gluconeogenesis to aid in the rapid metabolism of carbohydrate, fat and protein, nutrients needed in stressful situations [36]. Cortisol can be measured in urine, feces and saliva samples in addition to blood samples. Blood and saliva samples have a short time-frame of minutes, while urine and feces can give a longer time-frame up to a day [37].

\section{Management of Pre-slaughter Stress}

\subsection{Pre-slaughter dietary supplement}

Several pre-slaughter supplements (Magnesium, Chromium Tryptophan, electrolyte, and Vitamin E) have been developed and investigated to reduce pre-slaughter stress. It has been shown that adding Magnesium to the diet can decrease the plasma concentration of cortisol and catecholamine during stress, decreasing muscle glycogen loss and enhance live weight gains in cattle [38].

Chromium is an essential mineral that plays a role in glucose metabolism. This mineral may increase glycogen deposition by increasing the efficiency of insulin. Supplemental chromium may aid in increasing glycogen reserves which may reduce the depletion of glycogen prior to slaughter [39].Tryptophan is the precursor for the important neurotransmitter serotonin. Serotonin is central to the regulation of a number of key biological functions including temperature regulation, arousal, pain sensitivity, feeding, sexual behavior, and aggression. Tryptophan supplements may moderate stress in animals by stimulating brain serotonin levels [40].

Sodium is the major determinant of extracellular and total body water, whereas potassium is the primary 
determinant of intracellular fluid volume. Both of these cations are lost during ante mortem stress [23]. pre-slaughter feeding of electrolytes solution (Nutricharge) to cattle has been shown to maintain neutral $\mathrm{pH}$ of the body (acid-base balance) electrolyte balance, improve carcass yield by reducing dehydration and weight loss, reduce the incidence of dark cutting in cattle but there were no effects on initial or final $\mathrm{pH}$, muscle color or muscle water-holding capacity [41]. Feeding high sugar supplements or a mixture of electrolytes, sugars, and amino acids during pre-slaughter and/or lairage has been also confirmed by this author in reducing stress. Another study by [42] showed that Vitamin E supplement in the diet reduce stress during transport of animals to slaughtered assessed by decreased heart rate and cortisol concentration in saliva after transport.

\subsection{Appropriate livestock facilities and best animal handling practice}

One of the most effective means of minimizing pre-slaughter stress is to ensure that the animal handling facilities in good condition to enable smooth and efficient movement of livestock and that the stock persons understand the principles of animal handling. Livestock should be handled always in quietly and calmly manner to prevent injuries to livestock and employees. Ensure livestock always-on feed and water watch for nails, loose boards, and other hazards that could tear the hide or cause a bruise. Ensuring the proper handling of animals before, during and after transport as well as at slaughterhouse can reduce stress level A combination of well-trained handlers and properly designed facilities will reduce stress on cattle and improve both handling efficiency and productivity of cattle [43]. Therefore proper cattle handling requires the right facilities, equipment, and attitudes.

\subsection{Selection for temperament}

Temperament in livestock can be defined in a several ways but the preferred definition here is that it is the behavioral expression of the fearfulness of an animal in response to a challenging situation. Animals that display less fearful behavior during routine handling and management are less likely to injure themselves and their handlers. Selection for improved temperament or less fearful cattle will result in a general reduction in the magnitude of the stress response which in turn, may lead to reduced stress-mediated losses in meat quality [44].

\section{Effect of Pre-slaughter Stress on Meat and Carcass Quality}

Meat and carcass quality are affected by many factors but pre-slaughter stress seems to be the most crucial factor. Pre- slaughter stress leads to behavioral and physiological changes in cattle before slaughter. The energy required for muscle activity in the live animal is obtained from sugars (glycogen) in the muscle. In the healthy and well-rested animal, the glycogen content of the muscle is high. After the animal has been slaughtered, the glycogen in the muscle is converted into lactic acid, and the muscle and carcass become firm (rigor mortis). This lactic acid is necessary to produce meat, which is tasteful and tender, of good keeping quality and good color. If the animal is stressed before and during slaughter, the muscle glycogen is depleted and the lactic acid level that develops in the meat after slaughter is reduced. This produces meat and meat products that have high ultimate $\mathrm{pH}$, tough meat with higher cooking loss, poor palatable, short shelf, abnormal in muscle color and high water retention capacity. Due to such characteristic consumers discriminate against meat products from such animals [23]

According to [7] pre-slaughter stress due to improper and poor handling of animals during farming, marketing, loading, transporting, unloading, lairaging and stunning can cause undesirable end quality of product such as carcass damage (bruising, hemorrhages, blood splash), low carcass yields, and total carcass loss. Carcass damages result, not only in an economic loss to the meat chain but also it is a strong indicator of poor animal welfare [43].

The effect of stresses such as feed and water deprivation on live weight are and carcass weight studied by many scholars. The main effect of fasting (Food and water deprivation) and transportation in beef cattle have been shown a reduction in live weight of animals due to the reduction in gut fill and breakdown of muscle glycogen for energy [7] as well a loss in weight of carcass (warm carcass weight ) and edible offals [9]. Live animal weight loss also affected by ambient temperature, transport duration and type of cattle [45]. The losses in live or carcass weight refereed 'Shrinkage' which is a potential loss of yield of carcass and meat [9]. Carcass weight loss account up to one third of total live weight loss over the first $24 \mathrm{hr}$ of fasting and up to one and half between 24 and 48 hours. The loss in carcass weight is largely attributing to loss of protein and to a lesser degree fat, rather than loss 
of water [2].

A study reviewed by [6] had shown that effect of withholding water, feed and then transported for short possible time to slaughter plant on live weight loss. Live weight was lost most rapid at the beginning and amounted to $10.6 \%$ at 48 hour, while carcass losses observable at 24 and at 48 hour were up to $6.5 \%$. It has estimated that pre slaughter stress reduces live value by as much as $\$ 2$ to $\$ 5$ per hundred weights for cattle. Long feed deprivation period by providing only water, the stomach content of ruminant becomes more watery, increase chance of head, tongue and carcass contamination either through regurgitation or through accidental cutting of the gut wall during dressing. Under a long period of water deprivation, the removal of hiding may become more difficult because of dehydration of the subcutaneous tissue or greater tearing of the underlying tissue and a poor surface carcass appearance may occur [6].

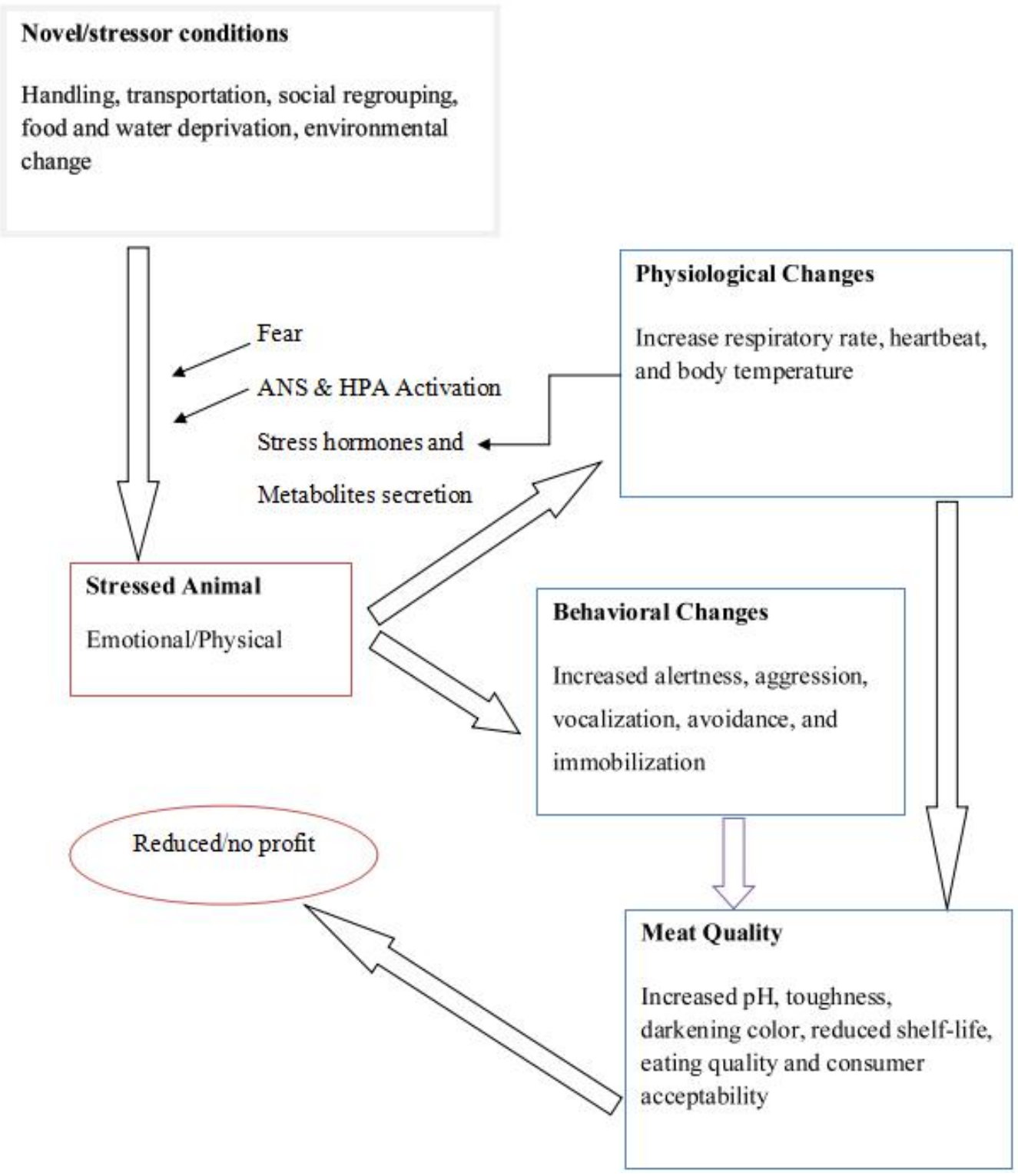

Figure 1 The effects of the pre-slaughter stress on the animals and the quality of meat (ANS-Autonomic Nervous System, HPA-Hypothalamic Pituitary adrenal).

\section{Conclusion}


Pre slaughter stress which affects the quality of meat depends on supply chain to which animal exposed from home to slaughter. There is a need to measure stress level along cattle and/or shoat supply chain using some enzymes, hormones and investigate their relationship with meat quality parameters in developing countries to improve the animal welfare condition and meat quality. Physiological stress measurement such as enzyme and hormone is not an easy task in developing countries as most of the time there is an inadequate analyzing machine, unavailability of reagents and long-distance of laboratory from sample collection site. Hence the researchers need to check the availability of these facilities. Ethiopia needs to improve livestock management (handling, transportation, feeding and, health care) in order to improve meat quality and enhance meat export.

\section{Conflict of Interest}

There was no conflict of interest regarding to this manuscript.

\section{References}

[1] Chambers, PG, Grandin T, Heinz G , Srisuvan, T (2004) Guidelines for Human Handling, Transport and Slaughter of Livestock. Published by Food and Agriculture Organization (FAO), Thailand.

[2] Warris, P.D (1995) Ante mortem factors influencing the yield and quality of meat from farm animals. PP 1-16. In: Jones S.D.M. (ed.), Quality and grading of carcasses of meat animals. CRC Press, Florida, USA.

[3] Von Holleben K, von Wenzlawowicz M, Gregory N, Anil H, Velarde A, Rodríguez P, Cenci Goga B, Catanese B and Lambooij B (2010) Report on good and adverse practices -animal welfare concerns in relation to slaughter practices from the viewpoint of $\quad$ veterinary $\quad$ sciences. http://rytualny.pl/data/uploads/pdf/dialrel-animal-welfare-during-slaughter-report-2010.pdf.

[4] Grandin, T (2007) Handling and Welfare of Livestock in Slaughter Plants, 3rd edition. CABI, Cambrige, MA, USA.

[5] Chulayo A.Y., Tada O and Muchenje V (2012) Research on pre-slaughter stress and meat quality: A review of challenges faced under practical conditions. Applied Animal Husbandry \& Rural Development, 5:1-6.

[6] Mareko, M.H (2005) Effect of Pre-slaughter Stress on Carcass or Meat Quality: Implication for Botswana Journal of Animal and Veterinary Advance, 4(9):761-767

[7] Adzitey, F (2011) Effect of pre-slaughter animal handling on carcass and meat quality: Mini Review. International Food Research Journal, 18: 484-490.

[8] Knowles, T. and Warriss, P (2007) Stress physiology of animals' during transport, 3rd edition. Cambridge business press, Boston, MA, USA.

[9] Warriss , P.D (2000) Meat science: An introductory text. CAB International Publishing, Wallingford, England.

[10] FAWC (Farm Animal Welfare Committee (2013).http://www.defra.gov.uk/fawc/about/five-freedoms

[11] Broom, D.M (2007) Causes of poor welfare and welfare assessment during handling and transport, pp. 1-14. In: Grandin Tempelte (ed.), Livestock Handling and Transport, CAB International, Wallingford, UK.

[12] Terlouw, E.M., Schouten, W.G., Ladewig, J (1997) pp 143-158. Physiology. In Appleby, M.C. and Hughes, B.O. (eds.), Animal Welfare. CAB International, Walhngford, UK.

[13] Dirbaba, D., and B. Hurrissa (2009) Live Animal Transport Services in Ethiopia: Current practices and future options. Norman Borlaug Institute for International Agriculture Working Paper 17th-TP-027-2009.Texas, US: College of Agriculture and Life Sciences at Texas A \&M University, Texas AgriLife Research, and the Texas AgriLife ExtensionService. http://borlaung.tamu.edu/files 2012/03/Live animal transport service in Ethiopia.

[14] Hall, S. J. and Bradshaw, R. H (1998) Welfare aspects of the transport by road of sheep and pigs. Journal of Applied Animal Welfare Science, 1(3):235-254.

[15] Knowles, T. G., Maunder, D. H. L. and Warriss, P. D (1994) Factors affecting the incidence of bruising in lambs arriving at one slaughterhouse. Veterinary Record, 134: 44-45.

[16] Ishiwata T, Uetake K, Eguchi Y and Tanaka, T (2008) Steer stress levels during long distance transport throughout the year in Japan. Journal of Animal Science, 79(4), 510-517.

[17] Sporer K.R, Weber P.S, Burton J.L, Earley B, Crowe M.A (2008) Transportation of young beef bulls alters circulating physiological parameters that may be effective biomarkers of stress. Journal of Animal Science, 86(6), 1325-1334

[18] Nijisane Y.Z and Muchenje V (2017) Farm to abattoir conditions, animal factors and their subsequent effects on cattle behavioral responses and beef quality. Asian and Australasian Journal of Animal Science, 30(6):755-764.

[19] Ohl F and VanderStaay, F.J (2012) Animal Welfare at the interface between science and society. The Veterinary Journal, 
192:13-19.

[20] Adzitey F and Nurul H (2011) Pale Soft Exudative (PSE) and Dark Firm Dry (DFD) Meats: Causes and measures to reduce these incidences. International Food Research Journal, 18:11-20.

[21] Grandin, T (2008) Engineering and design of holding yards, loading ramps and handling facilities for land and sea transport of livestock. Veterinaria italiana, 44(1): 235-245.

[22] Terlow E.M and Bourguet C.D (2012). Stress at slaughter in cattle: role of reactivity profile and environmental factors. Animal welfare, 21(2): 43-49.

[23] Ferguson, D and Warner, R (2008) Have we underestimated the impact of pre-slaughter stress on meat quality in ruminants? Meat Science, 80: 12-19.

[24] Warriss, P.D (2003) Optimal lairage times and conditions for slaughter pigs: Veterinary Record, 153(6):170-176

[25] Ponnampalam E.N, Hopkins D.L, Bruce H L, Gianluca, B and Bekhit, E.D (2017) Causes and contributing factors to dark cutting meat: Current Trends and Future Directions. Comprehensive review in food science and safety, 16:400-410.

[26] Armario, A (2006) The hypothalamic-pituitary-adrenal axis: what can it tell us about stressors? Central nervous system Neurological Disorder Drug Targets, 5(5): 485-501.

[27] Grandin, T (1999) Safe handlingof large animals. Occupational Medicine: State of the Art Reviews, 14: $195-212$.

[28] Mader, T.L (2003) Environmental stress in confined beef cattle. Journal of Animal Science 81(2), 110-119.

[29] Sattler, T and Furll, M (2004) Creatine kinase and aspartate aminotransferase in cows as indicators for endometritis. Journal Veterinary Medicine, 51, 132-137.

[30] Dieni CA, KB, Storey (2009) Creatine Kinase regulation by reversible phosphorylation in frog muscle. Comp. Biochem. Phys. (Part B) 152:405-412.

[31] Okeudo N.J and Moss B.W (2005) Serum Cortisol Concentration in Different Sex-Types and Slaughter Weights, and its Relationship with Meat Quality and Intramuscular Fatty Acid Profile. Pakistan Journal of Nutrition, 4 (2): 64-68.

[32] Minka N.S and Ayo J.O (2010) Physiological response of food animals to road transportation stress. African Journal of Biotechnology, 9: 6601-6613.

[33] Chulayo A, Muchenje V (2016). Activities of some stress enzymes as indicators of slaughter cattle welfare and their relationship with physico-chemical characteristics of beef. Animal, 1-8. doi:10.1017/S1751731117000222.

[34] Novak M.A., Hamel A.F.,Kelly B.J., Dettmer A.M and Meyer J.S (2013) Stress, the HPA axis, and nonhuman primate well-being: A review. Applied Animal Behaviour Science, 143:35-149.

[35] Gupta S, Earley B and Crowe M.A ( 2007) Effect of 12 hour road transportation on physiological, immunological and hematological parameters in bulbs housed at different space allowances. The Veterinary Journal, 173: 605-616.

[36] Mostl E and Palme R (2002) Hormones as indicators of stress. Domestric Animal Endocrinology, 23: 67-74.

[37] Novak M.A., Hamel A.F.,Kelly B.J., Dettmer A.M and Meyer J.S (2013) Stress, the HPA axis, and nonhuman primate well-being: A review. Applied Animal Behaviour Science, 143:35-149 [38] (Dunshea et al., 2005

[38] Hanson D, Calkins C, Milton T (2000) The effects of induced stress and supplemental chromium on meat quality of finishing heifers. Beef Cattle Report, 73: 82-83.

[39] Schaefer A.L, Dubeski P.L, Aalhus, J.L and Tong, A.K. (2001) Role of nutrition in reducing ante mortem stress and meat quality aberrations. Journal of Animal Science, 79(5):91-101.

[40] Schaefer A.L, Jones S.D and Stanley R.W (1997) The use of electrolyte solutions for reducing transport stress. Journal of Animal Science, 75:258-265.

[41] Peeters E, Neyt A, Beckers F, Desmet S, Aubert D and Geers R (2005) .Influence of supplemental magnesium, tryptophan, vitamin $\mathrm{C}$ and vitamin $\mathrm{E}$ on stress response of pigs to vibration. Journal of Animal science, 83(7):1568-1580.

[42] Grandin, T (2000) Livestock handling and transport, 2nd edition, CABI Publishing, Wallingford, Oxon, UK.

[43] King D.A., Pfeiffer C.E., Randel R.D., Welsh T.H., Oliphint R A and Baird, B.E (2006). Influence of animal temperament and stress responsiveness on the carcass quality and beef tenderness of feedlot cattle. Journal of Animal Science, 74(3):546-556.

[44] Gonzalez, L.A., Schwartzkopf-Genswein, K.S., Bryan, M., Silasi, R. and Brown, F (2012) Factors affecting body weight loss during commercial long haul transport of cattle in North America. Journal of Animal Science, 90:3630-3639. 